This item was submitted to Loughborough's Research Repository by the author.

Items in Figshare are protected by copyright, with all rights reserved, unless otherwise indicated.

\title{
Effect of supplementation of complex milk lipids in pregnancy on fetal growth: results from the Complex Lipids in Mothers and Babies (CLIMB) randomized controlled trial
}

\section{PLEASE CITE THE PUBLISHED VERSION}

https://doi.org/10.1080/14767058.2019.1683539

\section{PUBLISHER}

Informa UK Limited, trading as Taylor \& Francis Group

\section{VERSION}

AM (Accepted Manuscript)

\section{PUBLISHER STATEMENT}

This is an Accepted Manuscript of an article published by Taylor \& Francis in The Journal of Maternal-Fetal \& Neonatal Medicine on 19 November 2019, available online:

http://www.tandfonline.com/10.1080/14767058.2019.1683539.

\section{LICENCE}

CC BY-NC-ND 4.0

\section{REPOSITORY RECORD}

Norris, Tom, Renato Souza, Yinyin Xia, Ting Zhang, Angela Rowan, Sophie Gallier, Hua Zhang, Hongbo Qi, and Philip Baker. 2019. "Effect of Supplementation of Complex Milk Lipids in Pregnancy on Fetal Growth: Results from the Complex Lipids in Mothers and Babies (CLIMB) Randomized Controlled Trial". Loughborough University. https://hdl.handle.net/2134/11277926.v1. 
1 Effect of supplementation of complex milk lipids in pregnancy on fetal growth: results from the

2 Complex Lipids in Mothers and Babies (CLIMB) randomized controlled trial.

3 Tom Norris, Renato T Souza, Yinyin Xia, Ting Zhang, Angela Rowan, Sophie Gallier, Hua Zhang, Hong-

4 Bo Qi, Philip N Baker

5

6 School of Sport, Exercise and Health Sciences, Loughborough University, UK (TN)

7 Department of Health Sciences, University of Leicester, UK (TN, PNB)

8 Department of Obstetrics \& Gynaecology, University of Campinas, Brazil (RTS)

9 Department of Obstetrics and Gynaecology, the First Affiliated Hospital of Chongqing Medical

University, Chongqing, China ( $\mathrm{YX}, \mathrm{TZ}, \mathrm{HZ}, \mathrm{H}-\mathrm{BQ}, \mathrm{PNB})$

School of Public Health and Management; Research Center for Medicine and Social Development;

Innovation Center for Social Risk Governance in Health, Chongqing Medical University, Chongqing,

China (YX)

Fonterra Co-operative Group Ltd, Palmerston North, New Zealand (AR, SG)

Liggins Institute, University of Auckland, Auckland, New Zealand (PNB)

Disclosure statement: The design and conception of the study was led by the principal investigators.

The funders had no role in data collection and analysis or decision to publish.

\section{Conflict of Interest (COI) statement}

Angela Rowan and Sophie Gallier are employed by Fonterra Co-operative Group Ltd; the other authors have no financial conflicts of interest to disclose that may be relevant to this work.

Corresponding author:

Yinyin Xia, School of Public Health and Management, Chongqing Medical University, No. 1 Yixueyuan Road, Yuzhong District, Chongqing 400016, People's Republic of China. 


\section{Funding details}

This work was supported by the New Zealand Primary Growth Partnership postfarm gate dairy programme, funded by Fonterra Co-operative Group Ltd, New Zealand and the New Zealand Ministry for Primary Industries. Souza, R.T has been awarded a PhD scholarship from the CAPES Foundation, an agency under the Ministry of Education of Brazil, process 88881.134095/2016-01.

Short running title: No adverse effect of complex milk lipid supplementation on fetal growth parameters

Clinical trial registry: ChiCTR-IOR-16007700 (http://www.chictr.org.cn/enindex.aspx)

Data availability: The corresponding author confirms that we will make the data (in de-identified form) used in the manuscript, code book, and analytic code available to editors upon request either before or after publication for checking. 
Background: Gangliosides (GAs) are important for neuronal function and development of the brain, accumulating rapidly in the fetal brain during the last trimester of pregnancy. No study in humans has investigated whether maternal supplementation of GAs during pregnancy has an effect on fetal growth, particularly of the head circumference. Objective: To evaluate the effect of maternal dietary supplementation of complex milk lipids (CML; gangliosides and phospholipids) from the milk fat globule membrane (MFGM) during pregnancy on fetal growth. Design: Double-blind three-arm parallel randomized controlled trial of 1500 pregnant women from the Chongqing municipality of China, recruited between 11 and 14 weeks of pregnancy. Intervention was in the form of supplementation with: control maternal milk formulation containing a minimum of $2 \mathrm{mg}$ GA per serving ( 4 mg GA per day) vs a CML-enriched (CML-E) maternal milk formulation containing a minimum of $4 \mathrm{mg}$ GA per serving (8 mg GA per day) vs no maternal milk supplementation, but with standard obstetric care including prenatal folic acid supplementation. Main outcomes and measures were ultrasonographically-derived estimates of fetal growth in head circumference (HC) \& bi-parietal diameter (BPD) (primary outcomes); and abdominal circumference ( $A C)$, femur length (FL) and estimated fetal weight (EFW) (secondary outcomes) (Clinical trial registry: ChiCTR-IOR-16007700). Results: Supplementation with CML-E milk had no effects on size at mid-pregnancy or growth trajectories in any of the fetal biometric dimensions. Conclusions: Supplementation of CML from the MFGM from the end of the first trimester did not have any effects on fetal growth. The absence of any adverse growth outcomes suggests that maternal MFGM supplementation during pregnancy is safe and using CML-E milk formula can be a method of providing an increased GA and phospholipid supply in early life, which has been associated with neurodevelopmental benefits. 
Gangliosides (GAs) are a class of sialic acid-containing glycosphingolipids which are most abundantly expressed in the nervous system[1,2], particularly in the brain, where they constitute approximately $10 \%$ of the lipid matter of the neuronal membrane[3-5]. Gangliosides are critical for neuronal function and brain development and are implicated in such processes as neurotransmission, neurogenesis, synaptogenesis, cell proliferation and neuronal differentiation[3,6-9]. Phospholipids are critical constituents of cell membrane bilayers, providing structural fluidity and permeability. Phospholipids also supply precursors for the production of neurotransmitters and support myelination of the central nervous system and cognitive development (5).

During fetal development, there is a dramatic increase in brain ganglioside accretion at approximately 10-15 weeks gestation, which continues throughout gestation and into the postnatal period[10,11]. For example, Kracun et al[8] observed that between 16 and 22 weeks gestation, ganglioside concentration in the frontal cortex increased by $100 \%$, with a $30 \%$ increase in the hippocampus ${ }^{8}$. Fetal brain tissue during the earlier period of fetal development is predominantly composed of simple smaller gangliosides (e.g. GM3 and GD3), whereas towards the end of gestation and in the postnatal period, larger and more complex gangliosides predominate[12].

Two studies, one using pregnant rodents given intramuscular injection of gangliosides[13] and the other an ex vivo perfusion of human term placenta with gangliosides[12], suggested transfer of gangliosides across the placenta; in another rodent model, maternal dietary supplementation with complex milk lipids (CMLs) GAs and phospholipids during pregnancy and lactation results in an increased ganglioside concentration in the offspring brain, and also an increased brain weight two days after birth, suggesting an effect on fetal growth[14]. In a separate study investigating the effects of dietary supplementation of CML from the milk fat globule membrane (MFGM; as a source of gangliosides and phospholipids) in the immediate postnatal period in rats, an increased growth rate 
concentrations in human pregnancies are lacking. It is currently unknown whether a diet low or deficient in gangliosides (such as a vegan diet or dairy-free vegetarian diet) would have any detrimental impact on the development of the fetal brain or whether maternal GA synthesis is sufficient to support both her own health and the development of the fetus. In addition, we are not aware of any studies in humans which have investigated the effect of dietary ganglioside supplementation during pregnancy on fetal and infant growth rates.

The Complex Lipids in Mothers and Babies (CLIMB)[16] randomised controlled trial was initiated to investigate whether prenatal supplementation of gangliosides was associated with the primary outcome of maternal serum ganglioside levels. The secondary outcomes included birth outcomes (e.g. reductions in preterm birth, fetal growth restriction and pre-eclampsia) and infant cognitive development. Implicit within the secondary outcome of a reduction in fetal growth restriction is an association of ganglioside supplementation with improvements in fetal growth. Accordingly, we hypothesize that maternal dietary supplementation of gangliosides from the first trimester onwards will be associated with increases in ultrasonographically-derived estimates of fetal growth. In line with the observed functions of gangliosides, we hypothesise that effects will act primarily on estimates of fetal head growth (as a proxy for fetal brain development $[17,18]$ ), namely head circumference $(\mathrm{HC})$ and bi-parietal diameter (BPD), and these will therefore serve as the primary outcomes. We will also investigate the effects on other fetal dimensions for which we expect little or no effect (e.g. fetal abdominal circumference $(A C)$, femur length $(F L)$ and estimated fetal weight (EFW)), thus serving as negative controls. 
The CLIMB study is a randomized controlled trial (RCT) that recruited 1500 women attending their first prenatal care visit at either the First Affiliated Hospital of Chongqing Medical University (FCQMU) or Chongqing Health Centre for Women and Children (CHC), Chongqing, China. The detailed research protocol has been published elsewhere[16]. In brief, women aged 20-40 years with a singleton pregnancy were enrolled between 11-14 weeks of gestation. Recruitment began in September 2015 and ended in November 2016. Exclusion criteria were: allergy to milk or severe milk aversion, lactose intolerance and history of spontaneous or medically-indicated preterm birth before 32 weeks. Three clinical visits were performed during pregnancy (11-14 weeks, 22-28 weeks and 32-34 weeks) to collect maternal, nutritional, lifestyle, anthropometric and ultrasound data. Delivery and newborn data were obtained via medical records. Infants were followed up at 5 weeks and 12 months of age.

At the time of enrollment (11-14 weeks of gestation), women were randomized (1:1:1) into one of the three groups: 1) Control milk group: standard maternal milk formulation (product A) containing a minimum of $2 \mathrm{mg}$ GAs per serving (4 mg GAs per day); 2) CML-enriched (CML-E) group: milk formulation with a minimum of $4 \mathrm{mg} \mathrm{GAs} \mathrm{per} \mathrm{serving} \mathrm{(} 8 \mathrm{mg}$ of GAs per day); 3) Reference group: no maternal milk supplementation, but with standard obstetric care including prenatal folic acid supplementation. The CML-E maternal milk (Anmum ${ }^{\mathrm{TM}}$ maternal milk, Fonterra Co-operative Limited, New Zealand) was formulated with the ingredient SureStart ${ }^{\mathrm{TM}}$ MFGM Lipid 100 (supplied by NZMP, Fonterra Co-operative Limited), as a source of MFGM phospholipids and GAs (> $90 \%$ as GD3). MFGM gangliosides and phospholipids. 
The MedSciNet AB (Stockholm, Sweden) Interactive Web Response System generated random allocation for all participants to one of the three groups during the first study visit (11-14 weeks). Investigators, research nurses and the participants themselves knew if they had been allocated to the reference group (no intervention) or to a milk treatment group. However, where a participant was allocated to a milk formula, participants, research nurses and investigators were blinded as to which group they were in and which milk formula they received (participants received cans of milk of different colours and product codes, with the representation of each colour and code not known to either the research team or participant). Blinding was maintained until after analysis on the primary outcome was completed.

\section{Outcomes}

The primary outcomes in this study are trajectories of ultrasonographically-derived estimates of fetal $\mathrm{HC}$ and BPD. Secondary outcomes include fetal AC, FL and EFW. Fetal biometry was measured at approximately $11-14,22-28$ and 32-34 weeks gestation. Measurements were conducted in accordance with the Chinese Ultrasound Doctors Association's (CUDA) “Practice Guideline for the performance of Fetal Ultrasound Scan" (2012)[19].

Gestational age was estimated by last menstrual period (LMP) and was confirmed by ultrasound scan performed before 16 weeks. If there was either a discrepancy of seven or more days between LMP and ultrasound dating, or an unsure LMP, dates based on the early ultrasound scan were used.

The effect of the intervention was evaluated using an intention to treat (ITT) approach. Accordingly, trajectories were derived using mixed-effects models for all participants who contributed at least one measurement during gestation, under a missing at random (MAR) assumption ( $n=1481)$. An a priori decision was made to produce sex-specific growth trajectories which necessitated non-missing 
offspring sex data. This data was missing in 173 participants. As offspring sex was only collected at birth, the 173 with missing data represent a combination of i) those delivered at birth but without sex recorded, or ii) those not followed up at birth, as a result of either withdrawal from the study (although some women did consent for outcome data to be collected despite dropout), pregnancy termination or miscarriage. Table 1 shows the breakdown of terminations, miscarriages and withdrawals across the three treatment arms, in order to determine whether there were differential rates between groups. Baseline characteristics of the participants at recruitment stratified by intervention group, are presented in table 1 ( $n=1500$ ) and in supplemental table 1 for those who had fetal growth trajectories derived $(n=1308)$. The steps taken to arrive at this final sample are shown in figure 1 . Supplemental table 2 shows baseline characteristics of the 1308 in the final analysis vs the 192 (i.e. 1500-1308) with missing data.

The number of observations per participant ranged from 1-3, therefore fractional polynomials of one degree were fitted to the data, with the aim of identifying the most suitable age transformation to model the pattern of fetal anthropometry over gestation. In an attempt to stabilize the increasing variability in fetal size with gestational age, for a number of outcomes (HC, BPD, EFW) the modelling of fetal trajectories was done on the (natural) log-transformed dimensions, which has been advocated in previous studies of fetal growth[20-22]. After obtaining the best-fitting age transformation (based on fit statistics and inspection of residual plots), this was added as a fixed and random effect, allowing each participant to have their own individual growth trajectory. Subsequently, a fixed-effect for sex and its interaction with (transformed) gestational age were added to the models, in order to identify differences in size and growth between sexes. A final similar step was taken to investigate whether the intervention had any effect on size and growth over the gestational period. The mean group trajectories (control vs CML-E vs reference) were plotted to provide a visual representation of the effect of the intervention on each fetal biometric outcome. Where outcomes were log-transformed during the fitting process, these were back-transformed and plotted on their original scale. 
182 All analyses were conducted using StatalC v15 (Stata Corp, College Station, TX).

183 Ethics

184 The trial was approved by the local ethics committee of Chongqing Medical University (Ethic No.

185 2014034) and was conducted in accordance with the Declaration of Helsinki 1964 and the principles

186 of the International Conference on Harmonisation Good Clinical Practice E6 (ICH-GCP). Written

187 informed consent was obtained from all participants prior to recruitment. Confidentiality and

188 restricted access of individual participant data was assured. This trial was prospectively registered with

189 the Chinese Clinical Trial Register (ChiCTR-IOR-16007700). 
Table 1 provides baseline characteristics of women at recruitment $(n=1500)$, stratified by intervention group. Han Chinese was the predominant ethnic group (97.9\%) in the overall sample, which had a mean age of 28.4 years (SD: 3.5 ) and median body mass index of $21 \mathrm{~kg} / \mathrm{m}^{2}$ (inter-quartile range: 19.4; 22.9). Baseline characteristics across the three intervention groups were similar. Concordant values to these were observed in the final analysis sample overall $(n=1308)$ and within each intervention group in the final analysis sample (supplemental table 1), suggesting that there was no differential loss to follow-up between intervention arms.

\section{Primary outcomes}

Fetal HC

200

Gestational age ${ }^{\Lambda^{-1}}$ provided the best fitting transformation of the age scale. Model coefficients are presented in supplemental table 3 . Fetal sex was retained in the model and indicated that at the mean age (approximately 23 weeks), female $\mathrm{HC}$ was $1.2 \%$ (95\% Cl: $0.8 \%-1.5 \%)$ smaller than in males, however, there were no differences in the growth trajectory between the two sexes. The addition of the dummy variable for intervention group and its interaction with age ${ }^{\wedge-1}$ did not improve model fit and revealed no differences in size (at the mean age) and growth between the three intervention groups (supplemental table 3). HC trajectories (after back transformation) for the three intervention groups are presented in figures 2 and 3 , separately for each sex, with all three mean trajectory curves overlaid.

As per fetal $\mathrm{HC}$, the best fitting transformation of the age scale was gestational age $\mathrm{A}^{\wedge-1}$. BPD at the mean age (22.9 weeks) was also different between sexes, with females having a BPD which was 1.36\% (95\% Cl: $0.97 \%-1.75 \%)$ smaller than males, again with no sex differences observed in the BPD trajectory. The parameters in supplemental table 4 show that there was no effect of the intervention 
in either BPD size at the mean age or in BPD growth throughout pregnancy. Back transformed BPD trajectories for the three intervention groups are presented in figures 4 and 5 , separately for each sex.

\section{Secondary outcomes:}

Fetal AC

219

The best fitting fractional polynomial retained gestational age on the original scale (gestational age $\left.^{\wedge 1}\right)$. AC size at 22.9 weeks differed by sex, as shown by the sex dummy variable in supplemental table 5 , which reveals that $\mathrm{AC}$ was $1.13 \mathrm{~mm}$ smaller in females at this age. No differences were apparent in growth between sexes. The intervention had no effect on AC size (at the mean age) or growth trajectories (supplemental table 5) as evidenced by overlapping mean constant curves

224 (supplemental figures $1 \mathrm{a}$ and $1 \mathrm{~b}$ ).

Fetal FL

A transformation of gestational age ${ }^{\wedge 0.5}$ provided the best fit to the $\mathrm{FL}$ data. Unlike for HC, BPD and $\mathrm{AC}$, no difference in $\mathrm{FL}$ size was observed between sexes. FL did not differ between intervention groups in terms of size at 22.9 weeks or in FL trajectory over the course of gestation (supplemental table 6 and supplemental figure 2).

231 For estimated fetal weight the best fitting transformation of the age term was log age. At 22.9 weeks

232 females weighed $1.59 \%$ less than males ( $95 \% \mathrm{Cl}: 0.59 \%-2.58 \%)$, but there was no difference in EFW trajectories between sexes. Similarly, there were no differences in size or growth between intervention arms, as evidenced in supplemental table 7 and supplemental figures $3 \mathrm{a}$ and $3 \mathrm{~b}$. 
In this three-arm randomised controlled trial of 1500 pregnant women in Chongqing, we observed no adverse effects of a maternal milk supplement enriched with MFGM gangliosides and phospholipids on any of the fetal biometry trajectories.

With the observation that gangliosides play an important role in prenatal neuronal development within the central nervous system[10-12] and, in a rodent model, have been associated with an increased brain weight in the immediate postnatal period[14], we hypothesised that dietary supplementation with complex milk lipids, in particular gangliosides, would increase fetal growth in fetal head parameters, namely HC and BPD. There are various possible explanations for a lack of effect. One such explanation is that GD3, the principal ganglioside present in both milk intervention groups, may not be able to pass through the placenta into the fetal circulation. As was reported earlier, gangliosides have been observed to be able to cross the placenta, using both rat[13] and ex vivo human placental perfusion [12] models. However, in the former study, the ganglioside investigated was GM1 and was injected intramuscularly. In the latter study, while it was a mixture of dairy GD3 and GM3 that was investigated, it was observed that although there was significant uptake of both GD3 and GM3 on the maternal side and GM3 on the fetal side, there was only a 'non-statistically significant trend' for GD3 concentration to increase on the fetal side[12]. However, it is noted that GM3 is the major ganglioside in blood. Similarly, in the study by Gustavsson et al[14] which found an increased brain weight at birth in rats whose mothers had received dietary ganglioside supplementation during pregnancy, while an increased concentration of the major brain brain gangliosides GM1a, GD1b, GT1b was observed in the offspring brain, there was no increase in the concentration of GD3. However, as GD3 is a precursor of both GD1b and GT1b, the lack of an increase in GD3 may simply be indicative of its metabolism to the respective metabolites, which did show increases. A second possibility relates to the temporality of the metabolism of gangliosides during gestation. As has been shown elsewhere, GD3 (along with GM3) predominates in neuronal and glial precursor cells in earlier gestation[23,24], 
but in later gestation this shifts to other more complex gangliosides such as GM1 and GD1[12], which are metabolites of GD3 and GM3. As two of the three measurements used to produce our trajectories were taken in mid to late pregnancy, i.e. at a time when GD3 is predominantly metabolised into its downstream metabolites, it may be that the effects of supplementation of high concentrations of GD3 (on fetal growth, particularly of $\mathrm{HC}$ ) at this time may be limited by the capacity of the glycosyltransferases (ganglioside synthases) to metabolize GD3. Supplementation in mid to late gestation of the downstream metabolites of GD3 (i.e. GD1 or GT1) directly, which are therefore not dependent on the functioning of the intermediate glycosyltransferases may have generated different results. A further possibility is that the enrichment in the CML-E milk was not sufficient in order to elicit an effect on fetal growth. While one human study of supplementation of dietary gangliosides did report a positive effect on the plasma content of GD3, the supplemented dose was much greater than that of our study[25]. It has also been shown that in rare conditions such as GM3 synthase deficiency, enteral ganglioside supplementation in childhood can have positive effects on growth and neurodevelopment[26]. Finally, another more obvious explanation is that despite their correlation with overall brain size $[17,18]$, fetal $\mathrm{HC}$ and $\mathrm{BPD}$ are not sensitive enough to allow for the detection of any effect of gangliosides on fetal neuronal development.

276 A strength of the study is the large sample size obtained from an ethnically homogenous population of non-smoking and non-alcohol consuming women aged 20-40 years from a single city in China. The sample was well phenotyped, with serial collection of maternal blood profiles (for assessment of blood lipids, glucose and micronutrients), dietary assessment, blood pressure and anthropometry during gestation. A further strength was that, as this was a three-arm RCT with a standard milk formula and an enriched milk formula (with equal calories to the standard milk formula), we were able to determine the specific effects of ganglioside enrichment, over and above any effects which may have been a result of the potential increased caloric intake (versus those in the reference group). 
This was an analysis of the intervention on a secondary outcome of the trial, with the primary trial outcome being maternal serum ganglioside levels. As such, the sample size calculation was based upon detecting a $25 \%$ difference in standard deviations in the primary outcome (with alpha set at $5 \%$ and power at $80 \%$ ). While we did not perform a power calculation based on inter-group differences in EFW, the confidence intervals for the intervention effect are relatively tightly clustered around the null, suggesting that the intervention effect is somewhere close to the null. One notable limitation is the lack of detailed data regarding participant compliance with the intervention. The participants were not instructed to exclude dairy products and dairy-containing foods from their diet and therefore it was not possible to measure compliance by methods such as blood marker analysis; we could only rely on the accuracy of daily consumption logs and product returns - the possibility that milk formula was not used as intended cannot be excluded. For example, mothers in both the standard and CML-E formula groups received an increase in their daily caloric intake of approximately $300 \mathrm{kcal}$. As several reviews have shown that balanced protein/energy supplementation in pregnancy increases fetal growth (using birth weight as a proxy) $[27,28]$ and reduces small-for-gestational age deliveries (suggesting an upward shift in the birthweight distribution)[29], we were surprised to observe no increase in any fetal growth parameters in these groups. However, as many of the reported studies had interventions in excess of $300 \mathrm{kcal}$ per day, it may be that the interventions used in our study did not provide enough calories to elicit a detectable increase in fetal growth. We excluded from the analyses those with missing data, mostly comprising those missing data for offspring sex. As sex was only recorded at birth, this represents a proxy variable for infants who did not survive to birth (e.g. terminations and miscarriages) or whose mothers withdrew before birth, therefore potentially introducing a selection bias. As is shown in supplemental table 2 , the 1308 women who were retained in the analysis were a more educated group of women compared to the 192 excluded, therefore biasing the sample to a more educated sample overall. However, as is shown in supplemental table 1, 

sample of 1308 women. A further limitation is that no study-specific training was given to sonographers and no study of inter- and intra-sonographer reliability was conducted.

Conclusion:

312 We observed no adverse effects of supplementation with CML on fetal HC, BPD or any of the other 313 fetal biometric trajectories. Our finding of no effect on fetal growth trajectories may reflect an 314 inadequate dose of supplemented gangliosides and further investigation is required to determine 315 whether an increased concentration of MFGM gangliosides and phospholipids elicits any effect on 316 growth outcomes. Taken together, we conclude that the in-utero supplementation of pregnant

317 women with CML may be a safe method of providing an increased ganglioside and phospholipid supply 318 to the offspring in early life, which has been associated with neurodevelopmental benefits in the 319 postnatal period. 


\section{Conflict of Interest (COI) statement}

322 Angela Rowan and Sophie Gallier are employed by Fonterra Co-operative Group Ltd; the other authors

323 have no financial conflicts of interest to disclose that may be relevant to this work.

\section{Author's contributions}

325 1) $\mathrm{HZ}, \mathrm{H}-\mathrm{BQ}, \mathrm{PNB}$ designed research (project conception, development of overall research plan, and study oversight); 2) YX, TZ, HZ conducted research (hands-on conduct of the experiments and data

327 collection); 3) SG, AR provided essential reagents or provided essential materials; 4) TN and RTS analyzed data or performed statistical analysis; 5) TN, RTS, SG, PNB wrote paper; 6) PNB had primary responsibility for final content. 
[1] Yu RK, Nakatani Y, Yanagisawa M. The role of glycosphingolipid metabolism in the developing brain. J. Lipid Res. [Internet]. 2009 [cited 2018 Oct 25];50:S440-S445. Available from: http://www.ncbi.nlm.nih.gov/pubmed/18845618.

[2] Wang B, Brand-Miller J. The role and potential of sialic acid in human nutrition. Eur. J. Clin. Nutr. [Internet]. 2003 [cited 2018 Oct 25];57:1351-1369. Available from: http://www.ncbi.nlm.nih.gov/pubmed/14576748.

[3] Ledeen RW, Yu RK. Gangliosides: structure, isolation, and analysis. Methods Enzymol. [Internet]. 1982 [cited 2018 Oct 25];83:139-191. Available from: http://www.ncbi.nlm.nih.gov/pubmed/7047999.

[4] Kracun I, Rosner H, Drnovsek V, et al. Gangliosides in the human brain development and aging. Neurochem. Int. [Internet]. 1992 [cited 2018 Oct 25];20:421-431. Available from: http://www.ncbi.nlm.nih.gov/pubmed/1304337.

[5] Posse de Chaves E, Sipione S. Sphingolipids and gangliosides of the nervous system in membrane function and dysfunction. FEBS Lett. [Internet]. 2010 [cited 2018 Oct 25];584:1748-1759. Available from: http://www.ncbi.nlm.nih.gov/pubmed/20006608.

[6] Kolter T. Ganglioside Biochemistry. ISRN Biochem. [Internet]. 2012 [cited 2018 Oct 26];2012:1-36. Available from: http://www.ncbi.nlm.nih.gov/pubmed/25969757.

[7] Chiavegatto S, Sun J, Nelson RJ, et al. A Functional Role for Complex Gangliosides: Motor Deficits in GM2/GD2 Synthase Knockout Mice. Exp. Neurol. [Internet]. 2000 [cited 2018 Oct 25];166:227-234. Available from: http://www.ncbi.nlm.nih.gov/pubmed/11085888.

[8] Kracun I, Rosner H, Drnovsek V, et al. Human brain gangliosides in development, aging and disease. Int. J. Dev. Biol. [Internet]. 1991 [cited 2018 Oct 25];35:289-295. Available from: http://www.ncbi.nlm.nih.gov/pubmed/1814411.

[9] Yu RK, Tsai Y-T, Ariga T. Functional Roles of Gangliosides in Neurodevelopment: An Overview of Recent Advances. Neurochem. Res. [Internet]. 2012 [cited 2018 Oct 25];37:1230-1244. Available from: http://www.ncbi.nlm.nih.gov/pubmed/22410735.

[10] Svennerholm L, Boström K, Fredman P, et al. Human brain gangliosides: developmental changes from early fetal stage to advanced age. Biochim. Biophys. Acta [Internet]. 1989 [cited 2018 Oct 25];1005:109-117. Available from: http://www.ncbi.nlm.nih.gov/pubmed/2775765.

[11] Vanier MT, Holm M, Ohman R, et al. Developmental profiles of gangliosides in human and rat brain. J. Neurochem. [Internet]. 1971 [cited 2018 Oct 25];18:581-592. Available from: http://www.ncbi.nlm.nih.gov/pubmed/5581573.

[12] Mitchell MD, Henare K, Balakrishnan B, et al. Transfer of gangliosides across the human placenta. Placenta [Internet]. 2012 [cited 2018 Oct 25];33:312-316. Available from: http://www.ncbi.nlm.nih.gov/pubmed/22225907.

[13] Hungund BL, Morishima HO, Gokhale VS, et al. Placental transfer of (3H)-GM1 and its distribution to maternal and fetal tissues of the rat. Life Sci. [Internet]. 1993 [cited 2018 Oct 25];53:113-119. Available from: http://www.ncbi.nlm.nih.gov/pubmed/8515685. 
[14] Gustavsson M, Hodgkinson SC, Fong B, et al. Maternal supplementation with a complex milk lipid mixture during pregnancy and lactation alters neonatal brain lipid composition but lacks effect on cognitive function in rats. Nutr. Res. [Internet]. 2010 [cited 2018 Oct 25];30:279289. Available from: http://www.ncbi.nlm.nih.gov/pubmed/20534331.

[15] Vickers MH, Guan J, Gustavsson M, et al. Supplementation with a mixture of complex lipids derived from milk to growing rats results in improvements in parameters related to growth and cognition. Nutr. Res. [Internet]. 2009 [cited 2018 Oct 25];29:426-435. Available from: http://www.ncbi.nlm.nih.gov/pubmed/19628110.

[16] Huang S, Mo T-T, Norris T, et al. The CLIMB (Complex Lipids In Mothers and Babies) study: protocol for a multicentre, three-group, parallel randomised controlled trial to investigate the effect of supplementation of complex lipids in pregnancy, on maternal ganglioside status and subsequent cognitive outcomes in the offspring. BMJ Open [Internet]. 2017 [cited 2018 Oct 25];7:e016637. Available from: http://www.ncbi.nlm.nih.gov/pubmed/29025835.

[17] Cole TJ. Growth and Organ Development. Breast-Feeding Early Influ. Later Heal. [Internet]. Dordrecht: Springer Netherlands; [cited 2018 May 25]. p. 1-13. Available from: http://link.springer.com/10.1007/978-1-4020-8749-3_1.

[18] Cooke RW, Lucas A, Yudkin PL, et al. Head circumference as an index of brain weight in the fetus and newborn. Early Hum. Dev. [Internet]. 1977 [cited 2018 Oct 25];1:145-149. Available from: http://www.ncbi.nlm.nih.gov/pubmed/617306.

[19] Chinese Ultrasound Doctors Association. Practice guideline for the performance of fetal ultrasound scans. Chinese J. Med. Ultrasound. 2012;9.

[20] Owen P, Donnet ML, Ogston SA, et al. Standards for ultrasound fetal growth velocity. BJOG An Int. J. Obstet. Gynaecol. [Internet]. 1996 [cited 2018 May 25];103:60-69. Available from: http://doi.wiley.com/10.1111/j.1471-0528.1996.tb09516.x.

[21] Drooger JC, Troe JWM, Borsboom GJJM, et al. Ethnic differences in prenatal growth and the association with maternal and fetal characteristics. Ultrasound Obstet. Gynecol. [Internet]. 2005 [cited 2018 May 25];26:115-122. Available from: http://doi.wiley.com/10.1002/uog.1962.

[22] Royston P. DIY fractional polynomials. UK Stata User Gr. Meet. 2010.

[23] Goldman JE, Hirano M, Yu RK, et al. GD3 ganglioside is a glycolipid characteristic of immature neuroectodermal cells. J. Neuroimmunol. [Internet]. 1984 [cited 2018 Oct 25];7:179-192. Available from: http://www.ncbi.nlm.nih.gov/pubmed/6392333.

[24] Sbaschnig-Agler M, Dreyfus H, Norton WT, et al. Gangliosides of cultured astroglia. Brain Res. [Internet]. 1988 [cited 2018 Oct 25];461:98-106. Available from: https://www.sciencedirect.com/science/article/pii/0006899388907287.

[25] Miklavcic JJ, Shoemaker GK, Schnabl KL, et al. Ganglioside Intake Increases Plasma Ganglioside Content in Human Participants. J. Parenter. Enter. Nutr. [Internet]. 2017 [cited 2018 Oct 25];41:657-666. Available from: http://www.ncbi.nlm.nih.gov/pubmed/26673692.

[26] Wang $H$, Sency V, McJarrow P, et al. Oral ganglioside supplement improves growth and development in patients with ganglioside GM3 synthase deficiency. JIMD Rep. Springer; 2019. p. 9-20. 
[27] Kramer MS, Kakuma R. Energy and protein intake in pregnancy. In: Kramer MS, editor. Cochrane Database Syst. Rev. [Internet]. Chichester, UK: John Wiley \& Sons, Ltd; 2003 [cited 2018 Oct 25]. p. CD000032. Available from: http://www.ncbi.nlm.nih.gov/pubmed/14583907.

[28] Liberato SC, Singh G, Mulholland K. Effects of protein energy supplementation during pregnancy on fetal growth: a review of the literature focusing on contextual factors. Food Nutr. Res. [Internet]. 2013 [cited 2018 Oct 25];57:20499. Available from: http://www.ncbi.nlm.nih.gov/pubmed/24235913.

[29] Villar J, Merialdi M, Gülmezoglu AM, et al. Nutritional Interventions during Pregnancy for the Prevention or Treatment of Maternal Morbidity and Preterm Delivery: An Overview of Randomized Controlled Trials. J. Nutr. [Internet]. 2003 [cited 2018 Oct 25];133:1606S-1625S. Available from: http://www.ncbi.nlm.nih.gov/pubmed/12730475. 
Table 1 Baseline characteristics of women randomized into study $(n=1500)$

\begin{tabular}{|c|c|c|c|c|}
\hline & $\begin{array}{l}\text { Control milk } \\
\qquad(n=500)\end{array}$ & $\begin{array}{l}\text { CML-E milk } \\
\qquad(n=500)\end{array}$ & $\begin{array}{c}\text { Reference } \\
\text { group }(n=500)\end{array}$ & $\begin{array}{c}\text { Total } \\
(n=1500)\end{array}$ \\
\hline \multicolumn{5}{|l|}{ Maternal characteristics } \\
\hline Ethnicity (Han Chinese) & $493(98.6 \%)$ & $482(96.4 \%)$ & 493 (98.6\%) & $1468(97.9 \%)$ \\
\hline Age (years) & $28.4(3.6)$ & $28.6(3.5)$ & $28.3(3.4)$ & $28.4(3.5)$ \\
\hline Weight (kg) & $53.3(49 ; 60)$ & $53(49 ; 58.8)$ & $53.8(49 ; 58.8)$ & $53.1(49 ; 59)$ \\
\hline Height (cm) & $159.5(4.5)$ & $159.6(4.4)$ & $159.6(4.6)$ & $159.6(4.5)$ \\
\hline $\mathrm{BMI}\left(\mathrm{kg} / \mathrm{m}^{2}\right)$ & $21(19.4 ; 22.9)$ & $21(19.3 ; 22.9)$ & $21(19.5 ; 22.9)$ & $21(19.4 ; 22.9)$ \\
\hline \multicolumn{5}{|l|}{ Gravidity } \\
\hline 1 & $232(46.4 \%)$ & $229(45.8 \%)$ & $235(47 \%)$ & $696(46.4 \%)$ \\
\hline 2 & $141(28.2 \%)$ & $147(29.4 \%)$ & $138(27.6 \%)$ & $426(28.4 \%)$ \\
\hline $3+$ & $127(25.4 \%)$ & $124(24.8 \%)$ & $127(25.4 \%)$ & $378(25.2 \%)$ \\
\hline \multicolumn{5}{|l|}{ Parity } \\
\hline 0 & $389(77.8 \%)$ & $398(79.6 \%)$ & $383(76.6 \%)$ & $1170(78 \%)$ \\
\hline 1 & $109(21.8 \%)$ & $101(20.2 \%)$ & $114(22.8 \%)$ & $324(21.6 \%)$ \\
\hline 2 & $2(0.4 \%)$ & $1(0.2 \%)$ & $3(0.6 \%)$ & $6(0.4 \%)$ \\
\hline Fertility treatment (yes) & $11(2.2 \%)$ & $2(0.4 \%)$ & $6(1.2 \%)$ & $19(1.27 \%)$ \\
\hline $\begin{array}{l}\text { Marital status (married or de } \\
\text { facto) }\end{array}$ & $498(99.6 \%)$ & 499 (99.8\%) & 499 (99.8\%) & $1496(99.7 \%)$ \\
\hline Years of schooling & $16(15 ; 16)$ & $16(15 ; 16)$ & $16(15 ; 16)$ & $16(15 ; 16)$ \\
\hline Tertiary education (yes) & $311(62.2 \%)$ & $322(64.4 \%)$ & $319(63.8 \%)$ & $952(63.5 \%)$ \\
\hline $\begin{array}{c}\text { Tobacco smoking during } \\
\text { pregnancy (yes) }\end{array}$ & 0 & 0 & 0 & 0 \\
\hline $\begin{array}{c}\text { Alcohol consumption during } \\
\text { pregnancy (yes) }\end{array}$ & $1(0.2 \%)$ & 0 & 0 & $1(0.2 \%)$ \\
\hline \multicolumn{5}{|l|}{ Loss to follow-up } \\
\hline Number terminations & $11(2.2 \%)$ & $7(1.4 \%)$ & $11(2.2 \%)$ & $29(1.93)$ \\
\hline Number misscariages & $4(0.8 \%)$ & $4(0.8 \%)$ & $4(0.8 \%)$ & $12(0.8 \%)$ \\
\hline Withdrawal & $48(9.6 \%)$ & $56(11.20 \%)$ & $42(8.4 \%)$ & $146(9.73 \%)$ \\
\hline
\end{tabular}


427

428

429

430

431

432

433

434

435

436

437

438

\section{Figure legends}

Figure 1. Flow diagram of sample selection

Figure 2. Fetal $\mathrm{HC}$ trajectories by intervention group in males

Figure 3. Fetal $\mathrm{HC}$ trajectories by intervention group in females

Figure 4. Fetal BPD trajectories by intervention group in males

Figure 5. Fetal BPD trajectories by intervention group in females 